\title{
Solubility of Carvedilol in Ethanol + Propylene Glycol Mixtures at Various Temperatures
}

\author{
Sahel Vahdati, ${ }^{\dagger}$ Ali Shayanfar, ${ }^{\dagger}$ Jalal Hanaee, ${ }^{\S, \|}$ Fleming Martínez, ${ }^{\perp}$ William E. Acree, Jr., \\ and Abolghasem Jouyban*,\$ \\ ${ }^{\dagger}$ Biotechnology Research Center, Tabriz University of Medical Sciences, Tabriz 51664, Iran \\ ${ }^{\S}$ Drug Applied Research Center and Faculty of Pharmacy, Tabriz University of Medical Sciences, Tabriz 51664, Iran \\ ${ }^{\ddagger}$ Students’ Research Committee, Tabriz University of Medical Sciences, Tabriz 51664, Iran \\ "School of Life Sciences, University of Bradford, Bradford BD7 1DP, U.K. \\ ${ }^{\perp}$ Grupo de Investigaciones Farmacéutico-Fisicoquímicas, Departamento de Farmacia, Universidad Nacional de Colombia, A.A. 14490, \\ Bogotá D.C., Colombia \\ \#Department of Chemistry, University of North Texas, Denton, Texas 76203-5070, United States
}

Supporting Information

ABSTRACT: Solubilities of carvedilol (CVD) in binary mixtures of (ethanol + propylene glycol (PG)) at 298.2, 303.2, 308.2, and $313.2 \mathrm{~K}$ are reported. The modified versions of the van't Hoff and Gibbs equations were used to calculate the thermodynamic properties (enthalpy $\left(\Delta H^{\circ}\right)$, entropy $\left(\Delta S^{\circ}\right)$, and Gibbs energy $\left(\Delta G^{\circ}\right)$ standard changes of solutions) for CVD dissolved in (ethanol (1) + PG (2)) mixtures from the solubility data. The solubility data of CVD in (ethanol (1) + PG (2)) at different temperatures were correlated using different mathematical models, i.e., the Jouyban-Acree model, a combination of the Jouyban-Acree model with the van't Hoff model, and two modified versions of the Jouyban-Acree model. Solubility data of seven drugs in (ethanol (1) + PG (2)) at different temperatures were used to develop a quantitative structure-property relationship model for predicting solubility in solvent mixtures. In addition, enthalpy-entropy compensation using $\Delta H^{\circ}$ vs $\Delta G^{\circ}$ and $\Delta H^{\circ}$ vs $T \Delta S^{\circ}$ which explains the mechanism of cosolvency at different temperatures was discussed.

\section{INTRODUCTION}

Solubility is one of the crucial physicochemical properties in different stages of drug discovery and development, ${ }^{1}$ and solvent mixing or cosolvency is a simple, efficient, most frequent and feasible solubilization method used in the pharmaceutical industry. ${ }^{2}$ Solvent mixing is used in formulation of drugs, ${ }^{3}$ crystal engineering and synthesis of cocrystals, ${ }^{4}$ extraction, ${ }^{5}$ and optimization of the mobile phase of highperformance liquid chromatography (HPLC) in pharmaceutical analysis. ${ }^{6}$ In addition solvent mixing with temperature alteration is a common method to change the solubility of compounds in crystallization studies. Propylene glycol (PG) and ethanol are common (widely used) cosolvents in the pharmaceutical industry, and their mixing is used to prepare different commercial formulations. ${ }^{3}$ Addition of the cosolvent (or cosolvents) increases the solubility of drugs in aqueous solvent mixtures, which is an important issue in developing liquid oral drug formulations containing low-water-soluble drugs. On the other hand, the solubility of drugs in nonaqueous mixtures could be used in pharmaceutical formulations such as soft gelatin capsules where water is not compatible with such formulations.

Carvedilol (CVD) is a nonselective $\beta$ and $\alpha_{1}$ blocker that is used in the management of hypertension, angina pectoris, and congestive heart failure. It also has antioxidant effects and more promising therapeutic applications. ${ }^{7}$ From a biopharmaceutical classification point-of-view, CVD is a class II drug with low solubility and high permeability. So an increasing solubility and dissolution rate for CVD is a useful strategy to improve its bioavailability. ${ }^{8}$ Moreover, preparation of liquid dosage forms (e.g., for pediatric oral use form) of CVD is recommended for formulation. ${ }^{9}$

Determination of a drug's solubility is a costly and timeconsuming process, and the trial-and-error approach is often used to optimize the composition of solvent mixtures for dissolving a desired amount of a drug in a given volume of solution. To address this issue, several mathematical models have been proposed in order to correlate and/or predict the solubility of drugs in solvent mixtures. In addition, modeling of solubility in solvent mixtures can help to understand the mechanism of solubility in solvent mixtures. ${ }^{10}$

The Jouyban-Acree model was developed for correlating and/or predicting the solubility of drugs in solvent mixtures. The model provides accurate mathematical descriptions for how the solubilities of drugs vary as a function of both temperature and solvent composition for binary and ternary solvent mixtures. The model for representing the solubility of a drug in a binary mixture is ${ }^{10}$

Received: September 15, 2013

Revised: October 29, 2013

Accepted: October 30, 2013

Published: October 30, 2013 


$$
\begin{aligned}
\ln x_{m, T}^{\text {sat }}= & m_{1} \ln x_{1, T}^{\text {sat }}+m_{2} \ln x_{2, T}^{\text {sat }} \\
& +\frac{m_{1} m_{2}}{T} \sum_{i=0}^{2} J_{i}\left(m_{1}-m_{2}\right)^{i}
\end{aligned}
$$

where $m_{1}$ and $m_{2}$ are the fractions of solvents 1 and 2 in the absence of the solute, $x_{1, T}^{\text {sat }}$ and $x_{2, T}^{\text {sat }}$ are the solubilities of the solutes in the monosolvents, i.e., PG and ethanol in this work, respectively, and $J_{i}$ are the constants of the model computed by no intercept regression analysis. ${ }^{11}$

Solubility data at different temperatures could be correlated by the van't Hoff equation ${ }^{12,13}$

$$
\ln x_{T}^{\text {sat }}=A+\frac{B}{T}
$$

and for a nonlinear relationship, a three parameter equation could also be used, ${ }^{12}$

$$
\ln x_{T}^{\text {sat }}=A+\frac{B}{T}+C \ln T
$$

where $A, B$, and $C$ are the model constants calculated using a least-squares method.

A combination of the Jouyban-Acree model and the twoparameter van't Hoff equation has been proposed to correlate the solubility of drugs in solvent mixtures at different temperatures, ${ }^{14}$

$$
\begin{aligned}
\ln x_{m, T}^{\mathrm{sat}}= & m_{1}\left(A_{1}+\frac{B_{1}}{T}\right)+m_{2}\left(A_{2}+\frac{B_{2}}{T}\right) \\
& +\frac{m_{1} m_{2}}{T} \sum_{i=0}^{2} J_{i}\left(m_{1}-m_{2}\right)^{i}
\end{aligned}
$$

and for wider temperature ranges where nonlinear solubility behavior is observed one uses a slightly expanded form,

$$
\begin{aligned}
\ln x_{m, T}^{\text {sat }}= & m_{1}\left(A_{1}+\frac{B_{1}}{T}+C_{1} \ln T\right) \\
& +m_{2}\left(A_{2}+\frac{B_{2}}{T}+C_{2} \ln T\right) \\
& +\frac{m_{1} m_{2}}{T} \sum_{i=0}^{2} J_{i}\left(m_{1}-m_{2}\right)^{i}
\end{aligned}
$$

that contains the three-parameter van't Hoff equation.

$\mathrm{Ma}$ and co-workers ${ }^{13}$ and Sun et al. ${ }^{15}$ used modified versions of eqs 4 and 5 to correlate the solubility data in solvent mixtures at different temperatures in which the model constants are calculated with regression analysis (with intercept). Sun and co-workers used the following model $^{15}$

$$
\begin{aligned}
\ln x_{m, T}^{\text {sat }}= & D_{1}+\frac{D_{2}}{T}+D_{3} m_{1}+D_{4} \frac{m_{1}}{T}+D_{5} \frac{m_{1}^{2}}{T}+D_{6} \frac{m_{1}{ }^{3}}{T} \\
& +D_{7} \frac{m_{1}{ }^{4}}{T}
\end{aligned}
$$

whereas $\mathrm{Ma}$ and co-workers ${ }^{13}$ proposed an alternative modification on eq 5 as

$$
\begin{aligned}
\ln x_{m, T}^{\mathrm{sat}}= & E_{1}+\frac{E_{2}}{T}+E_{3} \ln T+E_{4} m_{1}+E_{5} \frac{m_{1}}{T}+E_{6} \frac{m_{1}^{2}}{T} \\
& +E_{7} \frac{m_{1}^{3}}{T}+E_{8} \frac{m_{1}^{4}}{T}+E_{9} m_{1} \ln T
\end{aligned}
$$

where $D_{1}-D_{7}$ and $E_{1}-E_{9}$ are the constants of the models. Specific details regarding the derivation of eqs 6 and 7 from eqs 4 and 5 have been discussed earlier. ${ }^{13}$

Thermodynamic properties of drugs dissolved in solvent mixtures can provide valuable information regarding the solution process from a microscopic point of view. The standard enthalpy $\left(\Delta H^{\circ}\right)$, entropy $\left(\Delta S^{\circ}\right)$, and Gibbs free energy $\left(\Delta G^{\circ}\right)$ changes associated with the dissolution process can be computed using a modified version of the van't Hoff equation $^{16}$

$$
\ln x_{T}^{\mathrm{sat}}=-\frac{\Delta H^{\circ}}{R}\left(\frac{1}{T}-\frac{1}{T_{\mathrm{hm}}}\right)
$$

$T_{\mathrm{hm}}$, the mean harmonic temperature, is used in van' Hoff analysis. $T_{\mathrm{hm}}$ is calculated as

$$
T_{\mathrm{hm}}=\frac{n}{\sum_{i=1}^{n}\left(\frac{1}{T}\right)}
$$

where $n$ is the number of temperatures.

In eq $8 R$ is the universal gas constant having a value of 8.314 $\mathrm{J} \mathrm{K}^{-1} \mathrm{~mol}^{-1}$. Numerical values of $\Delta G^{\circ}$ were calculated using

$$
\Delta G^{\circ}=-R T_{\mathrm{hm}} \times \text { intercept }
$$

Finally, the $\Delta S^{\circ}$ values for the solution process were computed by combining the $\Delta H^{\circ}$ and $\Delta G^{\circ}$ values as follows:

$$
\Delta S^{\circ}=\frac{\Delta H^{\circ}-\Delta G^{\circ}}{T_{\mathrm{hm}}}
$$

Relative enthalpic $\left(\% \xi_{\mathrm{H}}\right)$ and entropic $\left(\% \xi_{\mathrm{TS}}\right)$ contributions of dissolving CVD in (ethanol (1) + PG (2)) mixtures were calculated by eqs 12 and 13 , respectively:

$$
\begin{aligned}
& \% \xi_{\mathrm{H}}=100 \frac{\left|\Delta H^{\circ}\right|}{\left|\Delta H^{\circ}\right|+\left|T \Delta S^{\circ}\right|} \\
& \% \xi_{\mathrm{TS}}=100 \frac{\left|T \Delta S^{\circ}\right|}{\left|\Delta H^{\circ}\right|+\left|T \Delta S^{\circ}\right|}
\end{aligned}
$$

The literature reveals that these quantities provide valuable information for understanding the molecular events involved in the dissolution and transfer processes of drugs in multicomponent systems. ${ }^{17,18}$ For example, in the case of aqueous solutions, the predominance of the entropic term is commonly attributed to hydrophobic hydration, whereas the enthalpic predominance is attributed to solute-solvent hydrogen bonding.

The objectives of the present study are 3-fold: (1) to measure the solubility data of CVD in (ethanol (1) + PG (2)) mixtures at different temperatures, (2) to correlate the experimental solubility data using different mathematical models, and (3) to calculate the thermodynamic characteristic of CVD dissolved in (ethanol (1) + PG (2)) mixtures.

\section{EXPERIMENTAL SECTION}

2.1. Materials. CVD (0.990 mass fraction purity) was purchased from Salehanchimi (Tehran, Iran) and was used as received. The samples of PG ( 0.995 mass fraction purity) and absolute ethanol ( 0.995 mass fraction purity) used to prepare the binary solvent mixtures were supplied by Scharlau Chemie (Barcelona, Spain). The sample of ethanol (0.935 mass fraction purity) used for dilution of the samples prior to spectrophoto- 
metric analysis was obtained from Jahan Alcohol Teb (Arak, Iran).

2.2. Apparatus and Procedures. The binary mixtures of (ethanol (1) + PG (2)) mixtures were prepared as mass fractions from 0.00 to 1.00 at 0.10 intervals. Various solubility determination procedures were reviewed in a recent work. ${ }^{19}$ The solubility was determined using the saturation shake-flask method of Higuchi and Connors. ${ }^{20}$ An excess amount of CVD dissolved in the solvent was placed on a shaker (Behdad, Tehran, Iran) and equilibrated in an incubator equipped with a temperature-controlling system at different temperatures with the uncertainty of $0.2 \mathrm{~K}$ (Nabziran, Tabriz, Iran). A period of 5 days was needed to achieve equilibrium at the given temperature. The solutions were filtered using hydrophilic Durapore filters $(0.45 \mu \mathrm{m}$, Millipore, Carrigtwohill, Ireland) and then diluted quantitatively with ethanol. The absorbance of the solutions were recorded at $284 \mathrm{~nm}$ using a UV-vis spectrophotometer (Beckman DU-650, Fullerton, CA, USA), and the molar concentrations were calculated using a UV absorbance calibration graph. Each experimental data point represents an average of at least three experimental measurements on different, independently equilibrated samples. Densities of the saturated solutions were determined using a $5 \mathrm{~mL}$ pycnometer. The molar solubilities of CVD expressed in moles per liter unit were converted to mole fraction solubilities by employing the densities of the solute saturated solutions.

2.3. Computational Methods. The solubility of CVD was mathematically represented by five solution models: the Jouyban-Acree model (i.e., eq 1), a combination of the Jouyban-Acree model with the van't Hoff equation (eqs 4 and 5), and two modified Jouyban-Acree models (eqs 6 and 7). Thermodynamic properties of solutions of (ethanol (1) + PG (2)) solvent mixtures were calculated using the modified van't Hoff equation according to eqs 8-13.

The mean percentage deviation (MPD) was calculated as an error criterion of different numerical analyses

$$
\mathrm{MPD}=\frac{100}{N}\left[\frac{x_{m, \mathrm{calcd}}-x_{m, \text { exptl }}}{x_{m, \text { exptl }}}\right]
$$

where $N$ is the number of data points in each set.

\section{RESULTS AND DISCUSSION}

3.1. Solubility of CVD in (Ethanol (1) + PG (2)) Mixtures at Different Temperatures and Fitting Using Different Solution Models. Mass fraction compositions of (ethanol (1) + PG (2)) solvent mixtures, experimental (in molar and mole fraction units), of CVD are reported in Table 1. The minimum solubility of CVD (0.0019) among the investigated solvent systems in this work is observed in neat PG at $298.2 \mathrm{~K}$, whereas the maximum solubility is obtained in (ethanol (1) + PG (2) (4:6)) at $313.2 \mathrm{~K}$. The solubility of carvedilol is increased in the solvent mixtures and reached a maximum value at a given temperature. In addition, increased temperature lead to enhanced solubility of CVD as would be expected from past observations regarding the solubility of crystalline solutes. Mean relative standard deviations (RSDs) of three repetitive experiments was $6.2 \pm 2.3 \%$. Linearity was observed between the logarithm of solubility vs $1 / T$ according to eq 2 for all solvent systems $(R>0.96)$. The reported solubility data extend the available database of solubility of pharmaceuticals in aqueous and nonaqueous solvent mixtures. $^{21}$
Table 1. Experimental Molar Solubility $\left(C_{M} \pm S D\right)$ and Their Mole Fraction Values $\left(x_{T, \text { exptl }}^{\text {sat }} \times 1000\right)$ for CVD Solubility in (Ethanol (1) + PG (2)) Mixtures at Different Temperatures

\begin{tabular}{|c|c|c|c|}
\hline ethanol mass fraction & $T / \mathrm{K}$ & $C_{\mathrm{M}} \pm \mathrm{SD}$ & $x_{m, T}^{\mathrm{sat}} \times 1000$ \\
\hline 1.0 & 298.2 & $3.45 \times 10^{-2} \pm 1.04 \times 10^{-3}$ & 2.06 \\
\hline 0.9 & 298.2 & $4.23 \times 10^{-2} \pm 3.57 \times 10^{-3}$ & 2.64 \\
\hline 0.8 & 298.2 & $4.57 \times 10^{-2} \pm 2.45 \times 10^{-3}$ & 2.95 \\
\hline 0.7 & 298.2 & $5.38 \times 10^{-2} \pm 5.23 \times 10^{-3}$ & 3.61 \\
\hline 0.6 & 298.2 & $5.56 \times 10^{-2} \pm 2.58 \times 10^{-3}$ & 3.85 \\
\hline 0.5 & 298.2 & $5.57 \times 10^{-2} \pm 2.06 \times 10^{-3}$ & 3.95 \\
\hline 0.4 & 298.2 & $6.38 \times 10^{-2} \pm 4.31 \times 10^{-3}$ & 4.64 \\
\hline 0.3 & 298.2 & $7.15 \times 10^{-2} \pm 4.69 \times 10^{-3}$ & 5.34 \\
\hline 0.2 & 298.2 & $7.05 \times 10^{-2} \pm 1.22 \times 10^{-3}$ & 5.33 \\
\hline 0.1 & 298.2 & $5.52 \times 10^{-2} \pm 3.99 \times 10^{-3}$ & 4.19 \\
\hline 0.0 & 298.2 & $2.52 \times 10^{-2} \pm 1.20 \times 10^{-3}$ & 1.91 \\
\hline 1.0 & 303.2 & $5.27 \times 10^{-2} \pm 4.16 \times 10^{-3}$ & 2.86 \\
\hline 0.9 & 303.2 & $5.20 \times 10^{-2} \pm 4.29 \times 10^{-3}$ & 3.23 \\
\hline 0.8 & 303.2 & $6.04 \times 10^{-2} \pm 3.38 \times 10^{-3}$ & 3.91 \\
\hline 0.7 & 303.2 & $6.46 \times 10^{-2} \pm 5.16 \times 10^{-3}$ & 4.31 \\
\hline 0.6 & 303.2 & $7.20 \times 10^{-2} \pm 3.87 \times 10^{-3}$ & 4.98 \\
\hline 0.5 & 303.2 & $6.20 \times 10^{-2} \pm 7.50 \times 10^{-4}$ & 4.36 \\
\hline 0.4 & 303.2 & $7.63 \times 10^{-2} \pm 4.53 \times 10^{-3}$ & 5.52 \\
\hline 0.3 & 303.2 & $7.83 \times 10^{-2} \pm 4.61 \times 10^{-3}$ & 5.81 \\
\hline 0.2 & 303.2 & $7.53 \times 10^{-2} \pm 6.80 \times 10^{-3}$ & 5.65 \\
\hline 0.1 & 303.2 & $5.92 \times 10^{-2} \pm 4.61 \times 10^{-3}$ & 4.48 \\
\hline 0.0 & 303.2 & $3.52 \times 10^{-2} \pm 3.40 \times 10^{-3}$ & 2.67 \\
\hline 1.0 & 308.2 & $6.23 \times 10^{-2} \pm 5.63 \times 10^{-3}$ & 3.77 \\
\hline 0.9 & 308.2 & $6.66 \times 10^{-2} \pm 6.52 \times 10^{-3}$ & 4.20 \\
\hline 0.8 & 308.2 & $7.79 \times 10^{-2} \pm 6.04 \times 10^{-3}$ & 5.13 \\
\hline 0.7 & 308.2 & $7.77 \times 10^{-2} \pm 5.55 \times 10^{-3}$ & 5.30 \\
\hline 0.6 & 308.2 & $9.14 \times 10^{-2} \pm 7.94 \times 10^{-3}$ & 6.45 \\
\hline 0.5 & 308.2 & $7.93 \times 10^{-2} \pm 2.61 \times 10^{-3}$ & 5.70 \\
\hline 0.4 & 308.2 & $8.64 \times 10^{-2} \pm 3.48 \times 10^{-3}$ & 6.34 \\
\hline 0.3 & 308.2 & $8.74 \times 10^{-2} \pm 2.30 \times 10^{-3}$ & 6.60 \\
\hline 0.2 & 308.2 & $8.08 \times 10^{-2} \pm 1.51 \times 10^{-3}$ & 6.17 \\
\hline 0.1 & 308.2 & $6.87 \times 10^{-2} \pm 6.04 \times 10^{-3}$ & 5.29 \\
\hline 0.0 & 308.2 & $6.30 \times 10^{-2} \pm 2.91 \times 10^{-3}$ & 4.90 \\
\hline 1.0 & 313.2 & $6.42 \times 10^{-2} \pm 3.68 \times 10^{-3}$ & 3.93 \\
\hline 0.9 & 313.2 & $7.70 \times 10^{-2} \pm 5.97 \times 10^{-3}$ & 4.94 \\
\hline 0.8 & 313.2 & $8.61 \times 10^{-2} \pm 3.17 \times 10^{-3}$ & 5.76 \\
\hline 0.7 & 313.2 & $8.67 \times 10^{-2} \pm 5.58 \times 10^{-3}$ & 6.00 \\
\hline 0.6 & 313.2 & $1.19 \times 10^{-1} \pm 5.14 \times 10^{-3}$ & 8.67 \\
\hline 0.5 & 313.2 & $1.01 \times 10^{-1} \pm 5.41 \times 10^{-3}$ & 7.45 \\
\hline 0.4 & 313.2 & $9.75 \times 10^{-2} \pm 8.54 \times 10^{-3}$ & 7.38 \\
\hline 0.3 & 313.2 & $9.28 \times 10^{-2} \pm 5.26 \times 10^{-3}$ & 7.15 \\
\hline 0.2 & 313.2 & $8.94 \times 10^{-2} \pm 7.64 \times 10^{-3}$ & 6.98 \\
\hline 0.1 & 313.2 & $8.17 \times 10^{-2} \pm 4.59 \times 10^{-3}$ & 6.45 \\
\hline 0.0 & 313.2 & $7.40 \times 10^{-2} \pm 4.27 \times 10^{-3}$ & 5.92 \\
\hline
\end{tabular}

The measured CVD solubilities were correlated using various solution models briefly described in the Introduction. The obtained quantitative structure-property relationship (QSPR) models after including statistically significant constants $(p<$ 0.05 ) are

$$
\begin{aligned}
\ln x_{m, T}^{\mathrm{sat}}= & m_{1} \ln x_{1, T}^{\mathrm{sat}}+m_{2} \ln x_{2, T}^{\mathrm{sat}} \\
& +m_{1} m_{2}\left[\frac{727.338( \pm 49.024)}{T}\right. \\
& \left.-\frac{393.297( \pm 129.833)\left(m_{1}-m_{2}\right)}{T}\right]
\end{aligned}
$$




$$
\begin{aligned}
& \ln x_{m, T}^{\mathrm{sat}}=m_{1}\left[-4.493( \pm 0.196)+\frac{-41.174( \pm 5.943)}{T}\right] \\
& +m_{2}\left[-4.361( \pm 0.196)+\frac{-40.707( \pm 5.943)}{T}\right] \\
& +m_{1} m_{2}\left[\frac{666.266( \pm 61.711)}{T}\right. \\
& -\frac{365.917( \pm 114.991)\left(m_{1}-m_{2}\right)}{T} \\
& \left.+\frac{471.395( \pm 222.680)\left(m_{1}-m_{2}\right)^{2}}{T}\right] \\
& \ln x_{m, T}^{\mathrm{sat}}=m_{1}(-10.367( \pm 0.646)+1.318( \pm 0.186) \ln T) \\
& +m_{2}(-10.232( \pm 0.646)+1.321( \pm 0.186) \ln T) \\
& +m_{1} m_{2}\left[\frac{666.560( \pm 60.113)}{T}\right. \\
& -\frac{366.032( \pm 112.015)\left(m_{1}-m_{2}\right)}{T} \\
& \left.+\frac{472.128( \pm 216.916)\left(m_{1}-m_{2}\right)^{2}}{T}\right] \\
& \ln x_{m, T}^{\mathrm{sat}}=-4.094( \pm 0.112)-\frac{54.991( \pm 3.746)}{T} \\
& +\frac{78.804( \pm 7.118) m_{1}}{T}-\frac{69.004( \pm 6.855) m_{1}^{2}}{T}
\end{aligned}
$$

$$
\begin{aligned}
\ln x_{m, T}^{\mathrm{sat}}= & -11.969( \pm 0.426)+1.769( \pm 0.117) \ln T \\
& +\frac{78.846( \pm 6.961) m_{1}}{T}-\frac{69.037( \pm 6.705) m_{1}{ }^{2}}{T}
\end{aligned}
$$

The standard error of the model constants are contained in parentheses as ( \pm values) immediately following the respective constant. Figure 1 illustrates the calculated solubilities using eqs

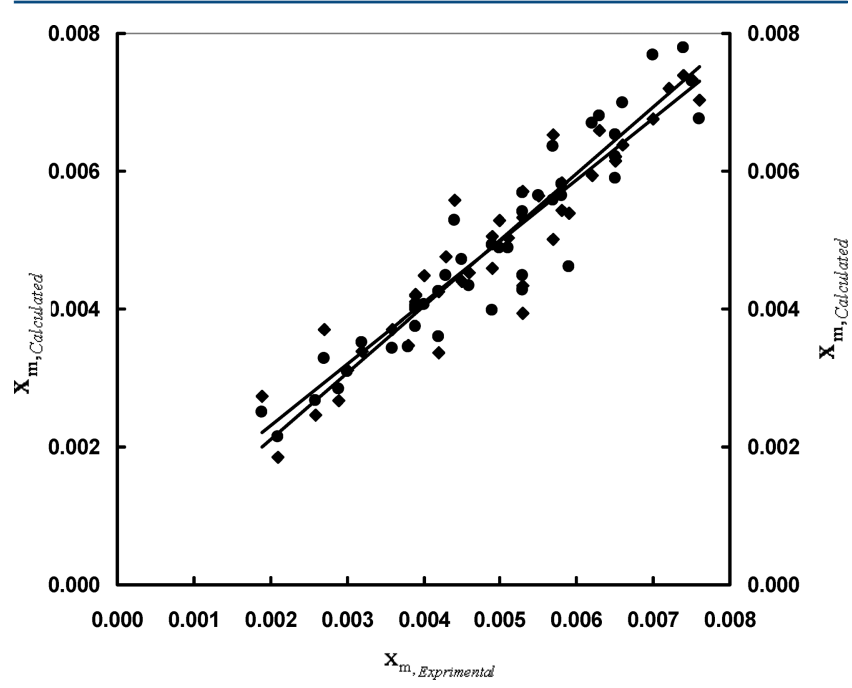

Figure 1. Plot of calculated mole fraction solubilities using eqs 15 ( and $16(\bullet)$ versus experimental values.
15 and 16 versus experimental values. The correlation coefficients of the calculated and experimental values for eqs 15 and 16 are 0.945 and 0.944 , revealing good fitness of the data.

Figure 2 shows the MPD values and their standard deviations. Equation 16 produced the most accurate model

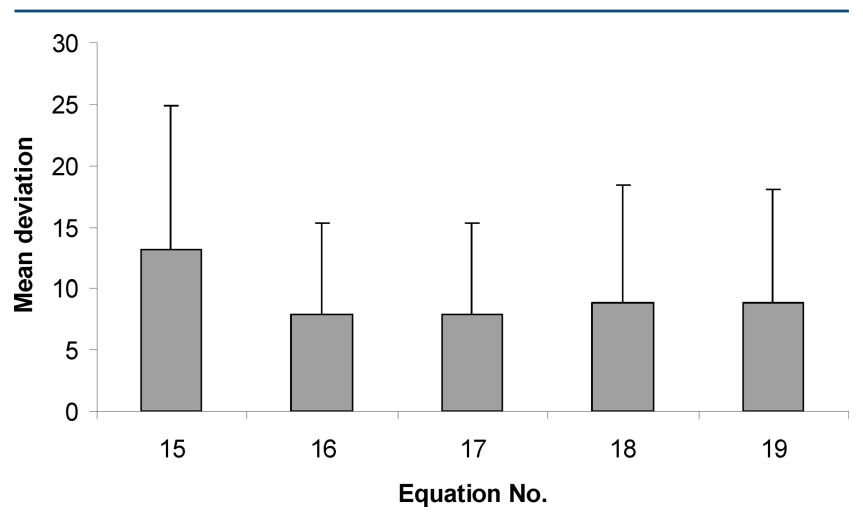

Figure 2. Mean deviations and their standard deviation for the correlated solubility data using various models.

among the investigated solution models. This finding is in agreement with the previous observations. ${ }^{22,23}$ The results of analysis of variance (ANOVA) reveal that the differences between MPDs of the investigated models are statistically significant $(p<0.0005)$. The ANOVA test recognizes the statistical significance of the mean values of more than two groups and the results reveal that at least one of the mean values is different from others. Further statistical analyses were conducted using Duncan's multiple test to recognize which model(s) is different from the others. The results of the Duncan test categorize the mean values in the subgroups where there is no significant difference between mean values within the subgroup. The results of the Duncan test indicated that MPD of eq 15 is different from other models and there are no significant differences between MPDs of eqs 16-19.

Overall, eq 4 affords several advantages over predictive/ descriptive eqs 5-7. First the model requires a minimum number of independent statistical significant parameters. Second, the model is simple both qualitatively and quantitatively, and according to the principle of Occam's razor, a good model does not increase beyond what is the necessary number of entities required to explain anything. Use of too many descriptors is one of the common errors in QSPR and quatitative structure-activity relationship (QSAR) studies. $^{24}$ Third, there is a theoretical justification for the model constants $\left(J_{i}\right)$ of eq $4 .{ }^{25}$

The Jouyban-Acree model could be trained using the solubility data of various solutes in a given binary solvent system as has been shown for aqueous mixtures of dioxane, ethanol, PG, and poly(ethylene glycol) 400 and the nonaqueous binary mixture of ethanol + ethyl acetate. ${ }^{10}$ Using these trained models, one could calculate the solubility of a drug in all solvent compositions of the binary solvents employing the experimental solubilities in the monosolvents, i.e., $x_{1, T}^{\text {sat }}$ and $x_{2, T}^{\text {sat }}$ A trained model proposed for describing the solubility of drugs in (ethanol (1) + PG (2)) mixtures employing the CVD data set and data sets collected from previous works is 


$$
\begin{aligned}
\ln x_{m, T}^{\mathrm{sat}}= & m_{1} \ln x_{1, T}^{\mathrm{sat}}+m_{2} \ln x_{2, T}^{\mathrm{sat}} \\
& +310.731( \pm 19.326)\left(\frac{m_{1} m_{2}}{T}\right)
\end{aligned}
$$

which is supported (associated) with a statistically significant $p$ value $(<0.0005)$ as well as $F$ value of 259 . The $F$ value is a function of the number of curve-fitting parameters of a model and the number of experimental data points ( $N$ in this work). The greater the $F$ value is, the less the probability value ( $p$ value), which is the more significant correlation. The backcalculated solubility data using eq 20 were compared with the corresponding experimental values and the obtained overall $\mathrm{MPD} \pm \mathrm{SD}$ was $12.2 \pm 12.5 \%$. Details of MPDs for the investigated drugs are listed in Table $1 S$ of the Supporting Information. There is no such a possibility for other models, i.e., eqs 4-7.

In addition, it is possible to train the Jouyban-Acree model using the solubility data measured at two different temperatures, usually the lowest and highest temperature of interest for each set, and calculate the solubility data in all solvent compositions and temperatures. The same computations were carried out using other solution models, and the obtained MPDs for eqs 2, 4, 5, 6, and 7 are 14.0, 9.7, 8.1, 8.3, and 8.9\%, respectively.

Overall, the results confirm that combination of the Jouyban-Acree model and the van't Hoff equation could be used to correlate the solubility of CVD in (ethanol (1) + PG (2)) mixtures at different temperatures; these results are in a good agreement with previous studies. ${ }^{14,26}$

3.2. Thermodynamic Parameters of CVD Solutions in (Ethanol (1) + PG (2)) Mixtures. Thermodynamic parameters including $\Delta H^{\circ}, \Delta S^{\circ}$, and $\Delta G^{\circ}$ of CVD solutions in (ethanol (1) + PG (2)) mixtures at $305.7 \mathrm{~K}$ (mean harmonic temperature) were computed using eqs $8-13 . \Delta H^{\circ}, \Delta S^{\circ}$, and $\Delta G^{\circ}$ in all solvent fractions are positive, and therefore, the dissolution process is endothermic and entropically driven. Relative contributions of enthalpy and entropy reveal that in all cases the main contributor to the standard Gibbs free energy of solution process of CVD is the enthalpy (for details, see Table $2 S$ of the Supporting Information).

3.3. Enthalpy-Entropy Compensation of Solution. The phenomenon of enthalpy-entropy compensation is widely applied in thermodynamic analyses of proteins, ligands, nucleic acids, and drug solubility. ${ }^{27,28}$ In the last case, this analysis is used to investigate the mechanism of solubilization at different temperatures, in particular the possible presence of hydrophobic hydration around nonpolar moieties of drugs dissolved in aqueous mixtures. ${ }^{29-32}$ In this study, plotting $\Delta H^{\circ}$ as a function of $T \Delta S^{\circ}$ and $\Delta H^{\circ}$ vs $\Delta G^{\circ}$ were used to assess enthalpy-entropy compensation for CVD solubility in (ethanol (1) + PG (2)) mixtures at $305.7 \mathrm{~K}$ (Figure 3). The linear plot $\left(r^{2}=0.999\right)$ of $\Delta H^{\circ}$ vs $T \Delta S^{\circ}$ with the slope approximately equal to 1.0 is illustrated for the solubility data in these solvent mixtures (Figure 3a). However, a nonlinear plot was observed for $\Delta H^{\circ}$ vs $\Delta G^{\circ}$ (Figure $3 \mathrm{~b}$ ). These results confirm previous studies that a linear profile between $\Delta H^{\circ}$ and $\Delta S^{\circ}$ cannot display the enthalpy-entropy compensation accurately because the range of $\Delta G^{\circ}$ measured in this study is much smaller than the range of $\Delta H^{\circ}$. Then, with respect to $\Delta H^{\circ}, \Delta G^{\circ} \approx$ constant (near to $13.5 \mathrm{~kJ} \mathrm{~mol}^{-1}$ ) and so linear $\Delta H^{\circ}-\Delta S^{\circ}$ compensation follows from $\Delta G^{\circ}=\Delta H^{\circ}-T \Delta S^{\circ} .{ }^{32,33}$ Thus, plotting of $\Delta H^{\circ}$ vs $\Delta G^{\circ}$ can be used to assess enthalpy-entropy compensation (a)
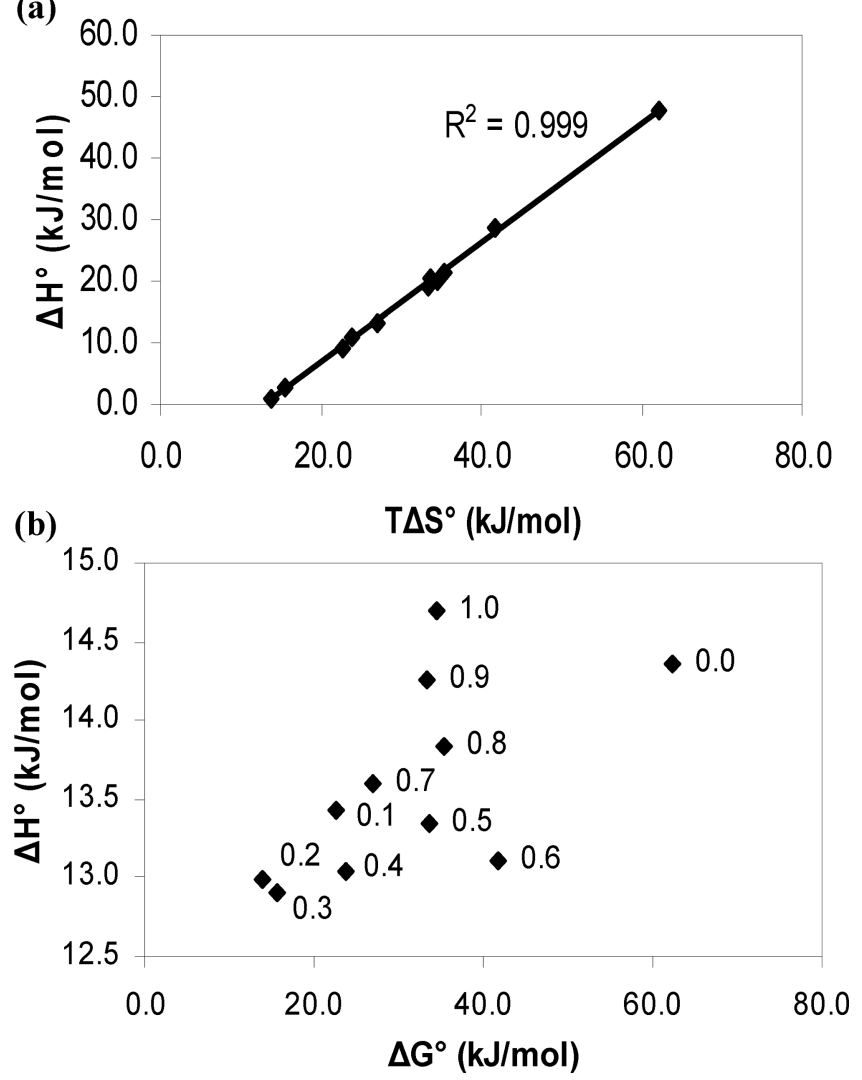

Figure 3. Enthalpy-entropy compensation plots: (a) $\Delta H^{\circ}$ vs $T \Delta S^{\circ}$ and (b) $\Delta H^{\circ}$ vs $\Delta G^{\circ}$ for solubility of CVD in (ethanol (1) + PG (2)) solvent mixtures at $305.7 \mathrm{~K}$.

and the effect of changing polarity of the medium on the solution process of the solute. The results show a complex and nonlinear profile with negative and positive slopes (if the composition points are connected by lines and the mass fraction of each cosolvent is varied by 0.10 ), indicating either entropy or enthalpy driving the solution processes according to the mixtures composition. According to the literature, negative slopes are associated with entropy-driven cosolvent effects whereas positive slopes correspond to cosolvent effects driven by the solution enthalpy. ${ }^{29-33}$ Nevertheless, because of the lack of information about the structural nature of (ethanol (1) + PG (20)) mixtures, explanations at the molecular level in terms of solute-solvent or solvent-solvent effect are not possible.

\section{CONCLUSION}

The solubility profile of CVD in (ethanol (1) + PG (2)) solvent mixtures shows a nonlinear pattern with maxima. Different models were applied to correlate solubility data of CVD in ethanol + PG. Combination of the Jouyban-Acree model with the van't Hoff equation can be used to correlate the solubility of CVD in (ethanol (1) + PG (2)) mixtures with significant parameters and with a better interpretation. In addition, the Jouyban-Acree model was applied to propose a QSPR model to calculate solubility drugs in (ethanol (1) + PG (2)) at different temperatures. It can also be concluded that the solution process of CVD in (ethanol (1) + PG (2)) mixtures is entirely dependent on the cosolvent composition. Nonlinear enthalpy-entropy compensation was found for CVD in the studied solvent system according to a plot of $\Delta H^{\circ}$ vs $\Delta G^{\circ}$. 


\section{ASSOCIATED CONTENT}

\section{S Supporting Information}

Tables listing the mean deviation for collected data from the literature and thermodynamic properties of the investigated solutions. This material is available free of charge via the Internet at http://pubs.acs.org.

\section{AUTHOR INFORMATION}

\section{Corresponding Author}

*E-mail: ajouyban@hotmail.com, jouyban@ut.ac.ir. Fax: +98 4113363231.

\section{Notes}

The authors declare no competing financial interest.

\section{ACKNOWLEDGMENTS}

This work is a part of the results of S.V.'s Pharm.D. thesis submitted to the Faculty of Pharmacy, Tabriz University of Medical Sciences, Tabriz, Iran. The authors acknowledge the Biotechnology Research Center, Tabriz University of Medical Sciences for providing partial financial support under Grant No. $5 / 85 / 848$.

\section{REFERENCES}

(1) Williams, H. D.; Trevaskis, N. L.; Charman, S. A.; Shanker, R. M.; Charman, W. N.; Pouton, C. W.; Porter, C. J. Strategies to Address Low Drug Solubility in Discovery and Development. Pharmacol. Rev. 2013, 65, 315-499.

(2) Rubino, J. T. Cosolvents and Cosolvency. Encyclopedia of Pharmaceutical Technology, 3rd ed.; Marcel Dekker: New York, 2006; pp 806-819.

(3) Strickley, R. G. Solubilizing Excipients in Oral and Injectable Formulations. Pharm. Res. 2004, 21, 201-230.

(4) Rager, T.; Hilfiker, R. Cocrystal Formation from Solvent Mixtures. Cryst. Growth Des. 2010, 10, 3237-3241.

(5) Kajdžanoska, M.; Petreska, J.; Stefova, M. Comparison of Different Extraction Solvent Mixtures for Characterization of Phenolic Compounds in Strawberries. J. Agric. Food. Chem. 2011, 59, 52725278.

(6) Pappa-Louisi, A.; Nikitas, P.; Papageorgiou, A. Optimisation of Multilinear Gradient Elutions in Reversed-Phase Liquid Chromatography Using Ternary Solvent Mixtures. J. Chromatogr., A 2007, 1166, 126-134.

(7) Sweetman, S. Martindale: The Complete Drug Reference; 34th ed.; Pharmaceutical Press: London, 2005.

(8) Liu, D.; Xu, H.; Tian, B.; Yuan, K.; Pan, H.; Ma, S.; Yang, X.; Pan, W. Fabrication of Carvedilol Nanosuspensions Through the AntiSolvent Precipitation-Ultrasonication Method for the Improvement of Dissolution Rate and Oral Bioavailability. AAPS PharmSciTech 2012, 13, 1-10.

(9) Buontempo, F.; Bernabeu, E.; Glisoni, R. J.; Quiroga, E.; Bregni, C.; Chiappetta, D. A. Carvedilol Stability in Paediatric Oral Liquid Formulations. Farm. Hosp. 2010, 34, 293-297.

(10) Jouyban, A. Review of the Cosolvency Models for Predicting Solubility of Drugs in Water-Cosolvent Mixtures. J. Pharm. Pharm. Sci. 2008, 11, 32-58.

(11) Jouyban-Gharamaleki, A.; Hanaee, J. A Novel Method for Improvement of Predictability of the CNIBS/R-K Equation. Int. J. Pharm. (Amsterdam, Neth.) 1997, 154, 245-247.

(12) Grant, D. J. W.; Mehdizadeh, M.; Chow, A. H. L.; Fairbrother, J. E. Non-linear van't Hoff Solubility-Temperature Plots and Their Pharmaceutical Interpretation. Int. J. Pharm. (Amsterdam, Neth.) 1984, $18,25-38$.

(13) Ma, H.; Qu, Y.; Zhou, Z.; Wang, S.; Li, L. Solubility of Thiotriazinone in Binary Solvent Mixtures of Water + Methanol and Water + Ethanol from (283 to 330) K. J. Chem. Eng. Data 2012, 57, 2121-2127.
(14) Jouyban, A.; Fakhree, M. A. A.; Acree, W. E., Jr. Comment on "Measurement and Correlation of Solubilities of (Z)-2-(2-Aminothiazol-4-yl)-2-methoxyiminoacetic Acid in Different Pure Solvents and Binary Mixtures of Water + (Ethanol, Methanol, or Glycol)". J. Chem. Eng. Data 2012, 57, 1344-1346.

(15) Sun, H.; Li, M.; Jia, J.; Tang, F.; Duan, E. Measurement and Correlation of the Solubility of 2,6-Diaminohexanoic Acid Hydrochloride in Aqueous Methanol and Aqueous Ethanol Mixtures. J. Chem. Eng. Data 2012, 57, 1463-1467.

(16) Panahi-Azar, V.; Shayanfar, A.; Martínez, F.; Acree, W. E., Jr.; Jouyban, A. Thermodynamic Studies of Fluphenazine Decanoate Solubility in Propylene Glycol + Water Mixtures and Correlation with the Jouyban-Acree Model. Fluid Phase Equilib. 2011, 308, 72-77.

(17) Perlovich, G. L.; Kurkov, S. V.; Kinchin, A. N.; Bauer-Brandl, A.. Thermodynamics of Solutions III: Comparison of the Solvation of (+)-Naproxen with other NSAIDs. Eur. J. Pharm. Biopharm. 2004, 57, 411-420.

(18) Perlovich, G. L.; Kurkov, S. V.; Bauer-Brandl, A. The Difference Between Partitioning and Distribution from a Thermodynamic Point of View: NSAIDs as an Example. Eur. J. Pharm. Sci. 2006, 27, 150157.

(19) Jouyban, A., Fakhree, M. A. A. Experimental and Computational Methods Pertaining to Drug Solubility. In Toxicity and Drug Testing; Acree, W. E., Jr., Ed.; InTech Publisher: New York, 2012.

(20) Higuchi, T.; Connors, K. A. Phase-Solubility Techniques. Adv. Anal. Chem. Instrum. 1965, 4, 117-212.

(21) Jouyban, A. Handbook of Solubility Data for Pharmaceuticals; CRC Press: Boca Raton, FL, USA, 2010.

(22) Jouyban-Gharamaleki, A.; Acree, W. E., Jr. Comment Concerning: Solubility Prediction of Caffeine in Aqueous $\mathrm{N}, \mathrm{N}$ Dimethylformamide Mixtures Using the Extended Hildebrand Solubility Approach. Int. J. Pharm. (Amsterdam, Neth.) 1999, 177, 127-128.

(23) Jouyban-Gharamaleki, A.; Valaee, L.; Barzegar-Jalali, M.; Clark, B. J.; Acree, W. E., Jr. Comparison of Various Cosolvency Models for Calculating Solute Solubility in Water-Cosolvent Mixtures. Int. J. Pharm. (Amsterdam, Neth.) 1999, 177, 93-101.

(24) Dearden, J. C.; Cronin, M. T.; Kaiser, K. L. How Not to Develop a Quantitative Structure-Activity or Structure-Property Relationship (QSAR/QSPR). SAR QSAR Environ. Res. 2009, 20, 241-266.

(25) Acree, W. E., Jr. Mathematical Representation of Thermodynamic Properties. Part 2. Derivation of the Combined Nearly Ideal Binary Solvent (Nibs)/Redlich-Kister Mathematical Representation from a Two-Body and Three-Body Interactional Mixing Model. Thermochim. Acta 1992, 198, 71-79.

(26) Jouyban, A.; Shayanfar, A.; Acree, W. E., Jr. Solubility Prediction of Polycyclic Aromatic Hydrocarbons in Non-Aqueous Solvent Mixtures. Fluid Phase Equilib. 2010, 293, 47-58.

(27) Sharp, K. Entropy-Enthalpy Compensation: Fact or Artifact? Protein Sci. 2001, 10, 661-667.

(28) Cornish-Bowden, A. Enthalpy-Entropy Compensation: A Phantom Phenomenon. J. Biosci. (New Delhi, India) 2002, 27, 121126.

(29) Bustamante, P.; Romero, S.; Peña, A.; Escalera, B.; Reillo, A. Enthalpy-Entropy Compensation for the Solubility of Drugs in Solvent Mixtures: Paracetamol, Acetanilide, and Nalidixic Acid in Dioxane-Water. J. Pharm. Sci. 1998, 87, 1590-1596.

(30) Gantiva, M.; Martínez, F. Thermodynamic Analysis of the Solubility of Ketoprofen in Some Propylene Glycol + Water Cosolvent Mixtures. Fluid Phase Equilib. 2010, 293, 242-250.

(31) Jiménez, J. A.; Martínez, F. Temperature Dependence of the Solubility of Acetaminophen in Propylene Glycol + Ethanol Mixtures. J. Solution Chem. 2006, 35, 335-352.

(32) Pacheco, D. P.; Manrique, Y. J.; Martínez, F. Thermodynamic Study of the Solubility of Ibuprofen and Naproxen in Some Ethanol + Propylene Glycol Mixtures. Fluid Phase Equilib. 2007, 262, 23-31. 
(33) Cantillo, E. A.; Delgado, D. R.; Martinez, F. Solution Thermodynamics of Indomethacin in Ethanol + Propylene Glycol Mixtures. J. Mol. Liq. 2013, 181, 62-67. 